\title{
Prognostic Value of Total Lesion Glycolysis in Stage III/ IV Small cell Lung Cancer
}

\author{
Huynh Quang Huy* \\ Pham Ngoc Thach University of Medicine and HCM Oncology Hospital, Vietnam
}

*Corresponding author: Huynh Quang Huy, Pham Ngoc Thach University of Medicine and HCMC Oncology Hospital. 2 Duong

Quang Trung street, District 10, Ho Chi Minh city, Vietnam

\section{ARTICLE INFO}

Received: 㓞 May 29, 2019

Published: 蔧 June 07, 2019

Citation: Huynh Quang H. Prognostic Value of Total Lesion Glycolysis in Stage III/IV Small cell Lung Cancer. Biomed J Sci \& Tech Res 18(4)-2019. BJSTR. MS.ID.003187.

Abbreviations: ${ }^{18} \mathrm{FDG} \quad$ PET-CT: $\left[{ }^{18} \mathrm{~F}\right]$ Fluoro-D-Glucose Positron-Emission Tomography; SCLC: Small Cell Lung Cancer; CCRT: Concurrent Chemoradiotherapy; $\mathrm{SUV}_{\max }$ : Maximum Standardized Uptake Value;OS: Overall Survival; TNM: Tumour Node Metastasis; TLG: Total Lesion Glycolysis; VALSG: Veterans Administration Lung Study Group
ABSTRACT

Objective: To investigate the prognostic and predictive importance of total lesion glycolysis (TLG) in patients with small cell lung cancer after concurrent chemoradiotherapy.

Methods: Forty patients with pathologically proven stage III and III SCLC had FDG PET-CT scans before concurrent chemoradiotherapy. The maximum standardized uptake value $\left(\mathrm{SUV}_{\max }\right)$ and TLG of the primary lung lesion was calculated. The relationship between the TLG and the long-term survival was studied after concurrent chemoradiotherapy.

Results: A total of 40 patients were analyzed and follow-up in 3 years. The mean of survival time was 12.6 months (95\%CI: 9.5 - 15.5 months). Only one case survived up to 36 months (3.1\%). The median of TLG of primary tumors was 207.98 , and patients were divided into higher $(\geq 207.98)$ and lower $(<207.98)$ TLG groups. The higher TLG group exhibited a significantly worse OS compared with the lower TLG group. Resession revealed a significant inverse relationship between TLG and affected survival rate.

Conclusions: The prediction of patients with stage III and IV SCLC is very poor. ${ }^{18} \mathrm{FDG}$ PET-CT is an effective method in the prediction of overall survival.

Keywords: Overall Survival; Small Cell Lung Cancer; Total Lesion Glycolysis (TLG)

\section{Introduction}

Lung cancer is the major cause of death in the developing countries, with an incidence of about 65-70 new cases per 100.000 [1]. Lung cancer is histologically divided into 2 main types: small cell lung cancer (SCLC) and non-small cell lung cancer (NSCLC). SCLC is an aggressive disease that accounts for approximately $14 \%$ of all lung cancers. Unlike NSCLC, in which major advances have been made using targeted therapies, there are still no approved targeted drugs for SCLC. Consequently, the 5-year survival rate remains low at $<7 \%$ overall, and most patients survive for only 1 year or less after diagnosis [2-4]. $\left[{ }^{18} \mathrm{~F}\right]$ fluoro-D-glucose positron-emission tomography $\left({ }^{18} \mathrm{~F}-\mathrm{FDG}\right.$ PET/CT) is widely used in lung cancer for staging, restaging and evaluation of the treatment response [5, 6]. Multiple studies demonstrate that PET/CT is more sensitive and specific than PET alone in evaluating the lung cancer since it provides combined morphological and functional information of the tumour [7-10].

High accuracy of PET/CT has been observed in the early assessment of response to therapy, showing a close correlation between the reduction of tumour metabolic activity measured after a course of therapy and the clinical outcome of patients after the previewed cycles of therapy in patients in advanced stage $[11,12]$. Tumour Node Metastasis (TNM) staging system, it is still the most reliable prognostic factor to predict the outcome. However, patients with SCLC may experience a worse outcome than expected. The prognostic and predictive importance of metabolic parameters such as SUVmax, metabolic tumor volume (MTV), 
and total lesion glycolysis (TLG) has been reported to predict the biologic aggressiveness of both early and advanced NSCLC [1316]; however, we do not find any prognostic studies for SCLC. The aim of this study was to investigate the prognostic and predictive importance of total lesion glycolysis (TLG) in patients with small cell lung cancer after concurrent chemoradiotherapy.

\section{Material and Methods}

\section{Clinical Data}

We prospectively analyzed the 18F-FDG PET-CT findings of 40 newly diagnosed SCLC patients between December 2010 and April 2019. They were selected according to the following criteria:

a) Pathologically proven stage III and IV SCLC

b) PET-CT was applied before any therapy.

These patients were followed up for 3 years. Patients was enrolled by convenient sampling method. The patients were referred to Bach Mai Nuclear Medicine and Oncology Center for initial staging with PET-CT scan and treated with CCRT. The tumor-node-metastasis (TNM) stage was determined according to the TNM $7^{\text {th }}$ edition [17]. TNM staging was obtained via information gathered through patient's chart including physical examination and total-body 18F-FDG PET/CT scan. Survival and death information were obtained from the hospitals databases and through phone calls to the patient families. The research proposal was approved by Institutional Review Board and Ethics Committee. The inclusion criteria were histologically proven SCLC, glycaemia lower than $140 \mathrm{mg} / \mathrm{dl}$ at the time of the exam, availability of FDG$\mathrm{PET} / \mathrm{CT}$ and tumour size $>20 \mathrm{~mm}$ to minimize the underestimation of SUV. Exclusion criteria were as follows:
a) Poor performance status
b) Diabetes (due to poor uptake of FDG)
c) Pregnancy.

\section{Concurrent Chemoradiation Therapy}

All patients were treated with CCRT. Chemotherapy consisting of $1-4$ cycles of cisplatin ( $20 \mathrm{mg} / \mathrm{m}^{2}$ ) given on days $1-5$ (or days $1-3$ ) and vinorelbine $\left(25 \mathrm{mg} / \mathrm{m}^{2}\right)$ given on days 1,8 , paclitaxel $(150 \mathrm{mg} /$ $\mathrm{m}^{2} \mathrm{~d}$ ) given on days 1,8 , or docetaxel $\left(75 \mathrm{mg} / \mathrm{m}^{2} \mathrm{~d}\right)$ given on days 1,8 . The first cycle of chemotherapy was applied the next day after the start of the radiotherapy. The second cycle of chemotherapy was applied 4 weeks after the first cycle. The radiotherapy was delivered by three-dimensional conformal radiotherapy technique. After setting up the patients in the vacuum bag, CT for treatment planning was performed in 4-mm slices, usually with intravenous contrast medium. Three-dimensional treatment planning was performed using the ADAC Pinnacle 7.4.

\section{FGD-PET-CT Imaging}

PET/CT imaging was performed with a median of 4 days (minimum 2 days, maximum 7 days) before starting treatment. Patients were asked to fast at least $6 \mathrm{~h}$ before the FDG-PET-CT scan. All patients had a glucose level below $180 \mathrm{mg} / \mathrm{dl}$ and were injected intravenously with $0.15-0.20 \mathrm{mCi} / \mathrm{kg}$ (7-12mCi) FDG. At 45-60 min after the injection, data were acquired from the vertex to the upper thigh. Immediately after CT, a PET scan (PET/CT Biograph True Point - Siemens, Germany) was performed for about $25 \mathrm{~min}$, with seven to eight bed positions and $3 \mathrm{~min} /$ position. PET images were reconstructed iteratively with CT data for attenuation correction, using an inline integrated Siemens Esoft Workstation system. Computerized tomography integrated positron emission tomography fusion images in transaxial, sagittal, and coronal planes were evaluated visually, and the SUVmax of lesions was obtained from transaxial images.

\section{Quantitative Assessment}

After determining the tumors, commercial software (Siemens Medical Solutions) was used for semi automatically represent the tumor borders with a threshold of $40 \%$ of the maximal SUV for lesions. We checked the results with the fused tomography slides to determine if the adjustment was needed. All MTV and SUV were determined by attenuation-corrected 18F-FDG PET images. The regions of interest (ROI) were specified as primary lesion and metastatic sites of the tracer uptake in targeted lesions. Tumor volumes were calculated with the exact threshold for the MTV, and TLG computation. The metabolic volumes of the primary tumor and total tumor (defined as the sum of the signal intensities of the primary tumor plus the metastatic lesions) were determined as "MTV.T" and "total MTV.WB", respectively. The TLG was calculated by the MTV multiplying the SUVmean [18].

\section{Statistical Analyses}

Continuous variables were summarized by mean and standard deviation, and categorical variables were summarized by frequency and percentage. Cox proportional hazard model was used to correlate continuous independent variables with survival. Survival functions of different populations were estimated by Kaplan-Meier estimator and compared by log-rank test. Multivariate Logistic resession was applied to assess the association between survival of patients and clinical factors. All analyses were performed by SPSS 20.0 (Chicago, Illinois, USA).

\section{Results}

The study included 40 patients. Average age was $61.3 \pm 9.5$ years (range 38-81). Male/female ratio was 9.7/1. The SUVmax ranged from 2.36 to 20.40 (mean 10.68 \pm 4.96 ). The median TLG was 207.98 (95\% CI 86.1 to 278.6). Positron emission tomography-computed tomography scan results are listed in Table 1. A PET stage of IV was 
assigned to $46.9 \%$ of patients. The mean of tumor size, SUVmax and the median of TLG in PET stage IV were significant higher than those in PET stage III respectively. The mean of survival time after first performing PET/CT was 12.6 months (95\%CI: 9.5 - 15.5 months). Only one case survived up to 36 months (3.1\%). Figure 1 shows survival stratified by PET stage. There was a statistically significantly correlation between PET stage and survival $(p=$ 0.012), with survival decreasing as PET stage increased. Although TLG is a continuous variable, we thought that establishing "highrisk" and "low-risk" groups, based on TLG values, would act as a useful reference for clinicians. Dichotomization of TLG values was based on the median values. Patients who had an TLG higher than 207.98 had worse survival than patients with an TLG less than 207.98 ( $p<0.05$ ) (Figure 2). (Figure 3-6) are the PET-CT images of patient with SCLC at stage IV, according to the TNM classification. Our analysis conducted controlling for the TLG and other factors, the Multivariate Logistic Resession revealed a significant inverse relationship between TLG and affected survival rate. The detailed data are shown in (Table 2).

Table 1: Positron Emission Tomography-Computed Tomography Scan Results.

\begin{tabular}{|c|c|c|c|}
\hline Variables & PET stage III & PET stage IV & P value \\
\hline $\mathrm{N}$ & 17 & 15 & \\
\hline $\begin{array}{c}\text { Tumor size, } \\
\text { mean }(\mathrm{cm})\end{array}$ & $2.61 \pm 0.88$ & $7.88 \pm 1.96$ & $<0.01$ \\
\hline SUVmax & $8.44 \pm 4.49$ & $13.21 \pm 4.29$ & 0.018 \\
\hline TLG (median) & 62.63 & 483.47 & 0.001 \\
\hline
\end{tabular}

Table 2: Multivariate Logistic Resession.

\begin{tabular}{|c|c|}
\hline Factor & P value \\
\hline Sex & 0.517 \\
\hline Age & 0.162 \\
\hline Stage & 0.429 \\
\hline Pretreatment SUV $_{\max }$ & 0.001 \\
\hline Pretreatment TLG & 0.001 \\
\hline
\end{tabular}

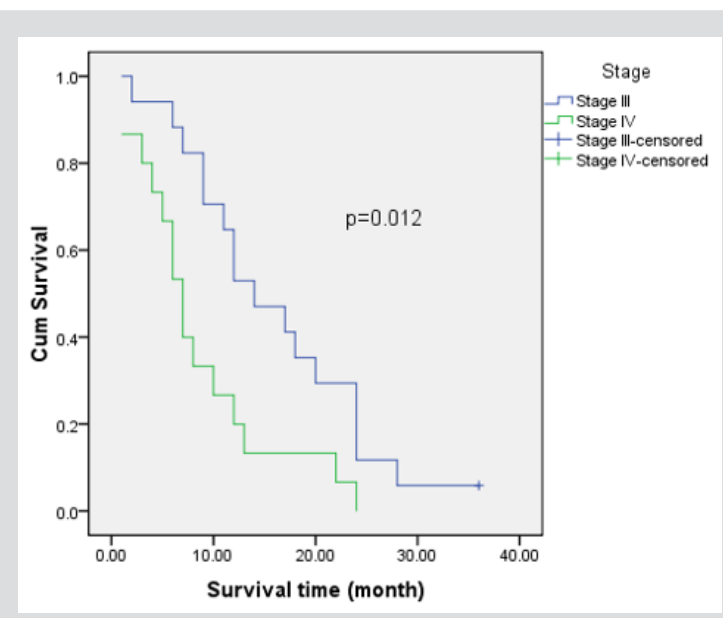

Figure 1: Positron emission tomography (PET) stage versus survival.

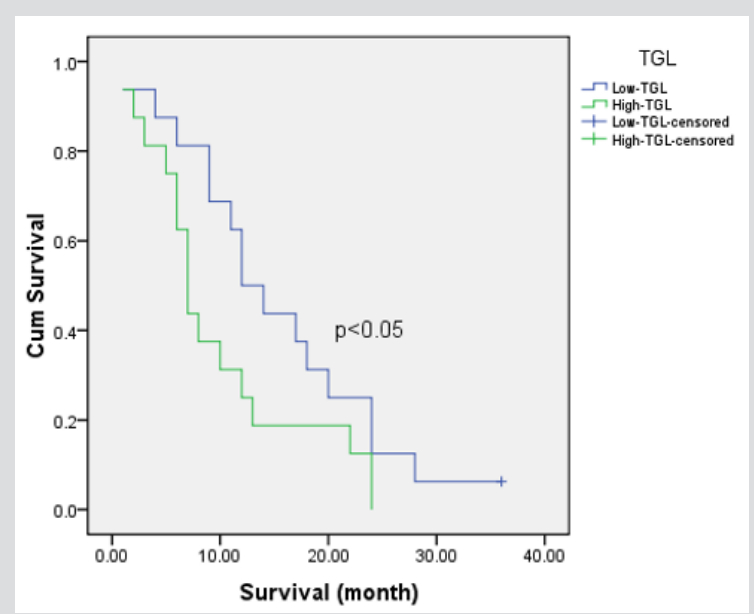

Figure 2: Total lesion glycolysis (TLG) versus survival.

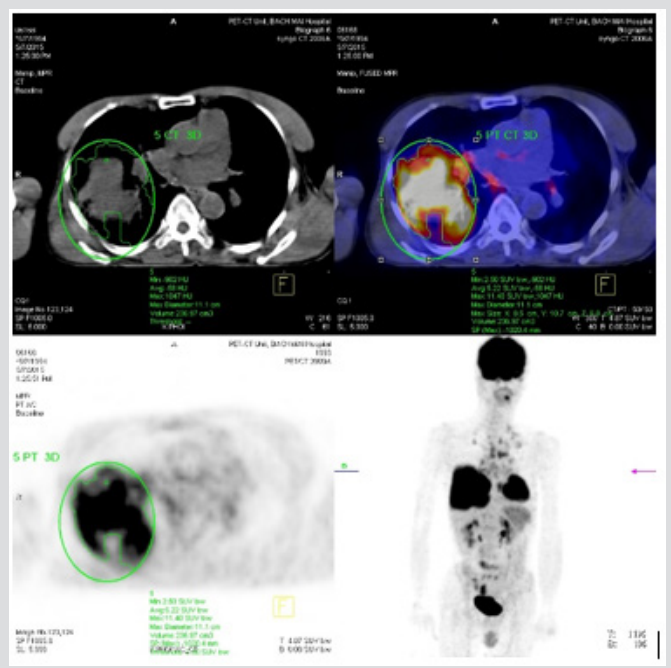

Figure 3: The primary tumor located at upper right lobe with tumor diameter was $11.1 \mathrm{~cm}$ and SUVmax was 11.40 .

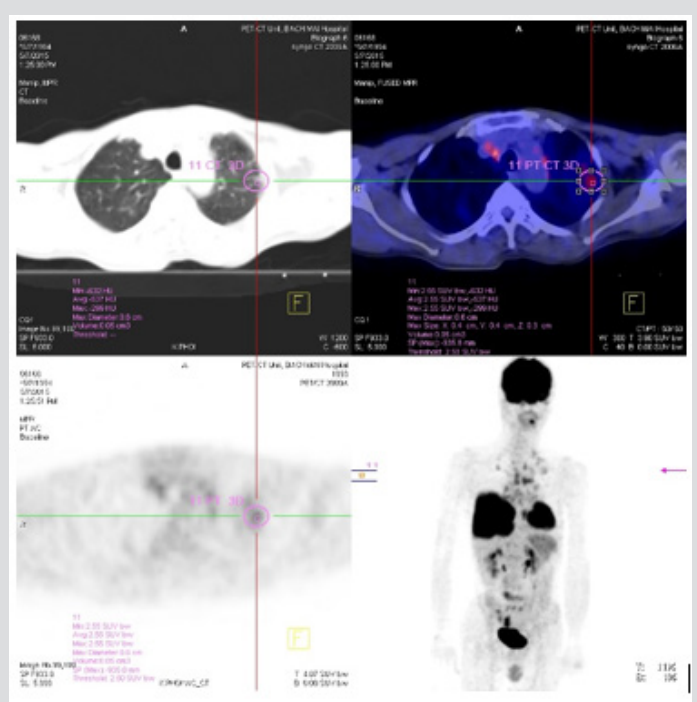

Figure 4: A leision located at upper left lobe was detected on PET/CT as lung metastasis with tumor diameter: 0.6 $\mathrm{cm}$ and SUVmax: 2.55 . 


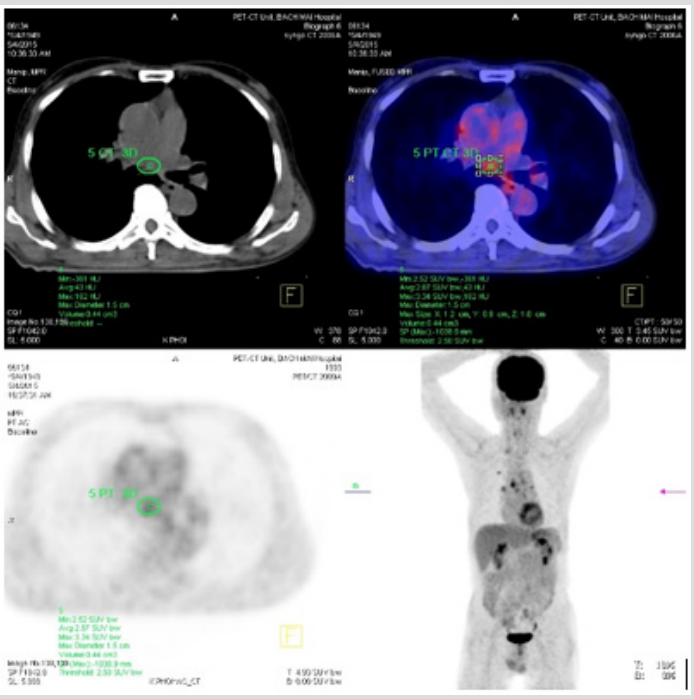

Figure 5: A mediastinal was detected by PET/CT as a metastasis leision with tumor diameter: $1.5 \mathrm{~cm}$ and SUVmax: 3.34 .

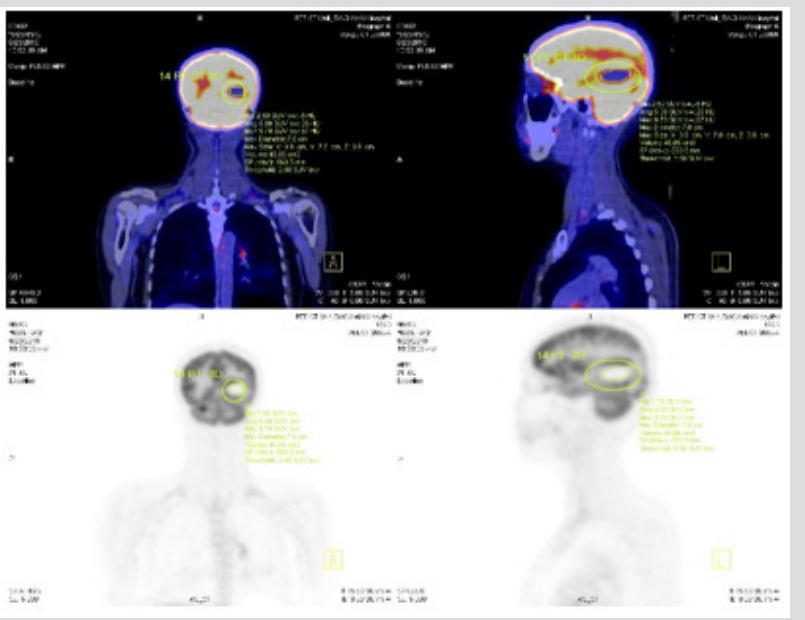

Figure 6: A SCLC patient with brain metastasis at right temporal lobe with tumor diameter was $7.0 \mathrm{~cm}$ and SUVmax was 9.7.

\section{Discussion}

Small cell lung cancer (SCLC) is a subtype of lung cancer with poor prognosis. It is estimated that nearly two million individuals are diagnosed as lung cancer every year, approximately $15 \%$ of which are SCLC [19]. SCLC is characterized by a rapid doubling time and the propensity for early dissemination. Chemotherapy remains the first line therapy for SCLC. Despite the initial response to chemotherapy, most tumors ultimately would develop drug resistance which is associated with the unsatisfied prognosis. Only $10-15 \%$ of patients with limited disease are still alive 2 years after diagnosis, while the overall survival (OS) of patients with extensive disease is even shorter $[20,21]$. All of patients in our study were at stage III and IV, so the survival time was within 36 months after first performing PET/CT. The mean of survival time was 12.6 months (95\%CI: 9.5 - 15.5 months). Only one case survived up to
36 months (3.1\%). Although CT or magnetic resonance imaging (MRI) provides precise anatomical and morphological information, the role of FDG-PET-CT has increased for diagnosis and staging of lung cancer [22]. Recently, FDG uptake has been reported to be a prognostic factor in patients with lung cancer [22-24]. Patz et al. [25] demonstrated that patients with positive FDG-PET-CT results, after treatment for lung cancer, had a significantly worse prognosis than patients with negative results. In recent years, metabolic tumor volume and tumor lesion glycolysis gives more information about tumor metabolic activity and showed promising outcome results in NSCLC patients $[26,27]$, but no similar data was found for those in SCLC

The goal of our study was to understand the ability of PET-CT scan to predict overall outcome. Our results show that PET-CT scan can in fact act as a prognosticator for long-term survival. There are many different aspects of PET-CT scan that were reviewed in this study. Overall PET stage was seen to predict survival in our study. This finding has been seen previously [28] and is in part related to the poor overall outcome in patients identified with advanced disease, especially in patients with M1 disease [29]. Because patients with M1 disease have such guarded outcomes, we performed separate analyses of the role of TLG versus survival excluding these patients. Even after excluding patients with M1 disease, there was still a significant correlation between TLG and survival. Importantly, these analyses were performed adjusting for mass size to prevent potential confounding from a variable already known to be associated with worse survival. These findings are important in that they can perhaps guide treatment plan based on these values, as the TLG levels are known pretreatment.

We also thought it was important to analyze the correlation of TLG with survival within each clinical stage. But it is not significant in this study because of small sample size. Our study has shown that survival decreases as TLG of the primary tumor increases. An important point that remains to be discovered, however, is the mechanism of failure in these patients. One potential mechanism is that tumors with higher TLG values have a more advanced stage at surgery than predicted by the pretreatment PET stage, implying that as the TLG increases, accuracy decreases. Another potential mechanism is earlier local recurrence of disease, implying that tumors with higher TLG values are more locally aggressive. Yet another possible mechanism is an increased propensity for distant metastasis. Prospective studies are required to determine the absolute causes for decreased survival in patients with higher TLG values.

Although we believe that TLG should be used as a gradient, we attempted to find a cutoff value, above and below which there were significant differences in survival. We were able to achieve this for TLG, with values of 207.98. We believe that these cutoff points can be useful as a reference for clinicians, and may eventually be able to be incorporated into a staging system. Further prospective studies 
are required, however, before this goal can be achieved. This cutoff would be especially practical in patients with no evidence of mediastinal disease pretreatment. Better ability to stratify these patients would lead to more accurate prediction of long-term outcome and more appropriate treatment preoperatively. Our results argue that patients with a high TLG would potentially profit from a more aggressive treatment plan, including mediastinoscopy before resection of the primary tumor and adjuvant chemotherapy, regardless of final pathologic results.

Many studies were on prediction of survival or treatment outcome in patients with NSCLC but we did not find any report of those in SCLC using Primary tumor standardized uptake value on 18F-FDG PET/CT. SCLC is a subtype of lung cancer associated with dismal prognosis. The $7^{\text {th }}$ TNM classification and VALSG staging system are the most widely used models to predict the clinical outcome of SCLC currently [30]. This study has some limitations because of the small sample size and all patients were at stage III and IV. Further studies with larger patient groups are needed to assess the relationship between primary tumor TLG and overall and disease-free survival in patients with SCLC.

\section{Conclusion}

In conclusion, a pretreatment TLG of $\geq 207.98$ exhibited a worse OS compared with those with an TLG $<207.98$ in SCLC patients. These results indicate that pretreatment TLG is a prognostic marker that could be used to identify high-risk patients with SCLC. Additional studies are warranted to determine if pretreatment TLG is associated with long-term prognosis.

\section{References}

1. Barta JA, Powell CA, Wisnivesky JP (2019) Global Epidemiology of Lung Cancer. Ann Glob Health 85(1).

2. Abdel Rahman $O$ (2018) Changing epidemiology of elderly small cell lung cancer patients over the last 40 years; a SEER database analysis. Clin Respir J 12(3): 1093-1099.

3. Yu JB, Decker RH, Detterbeck FC, Wilson LD (2010) Surveillance epidemiology and end results evaluation of the role of surgery for stage I small cell lung cancer. J Thorac Oncol 5(2): 215-219.

4. Zhang R, Li P, Li Q, Qiao Y, Xu T, et al. (2018) Radiotherapy improves the survival of patients with extensive-disease small-cell lung cancer: a propensity score matched analysis of Surveillance, Epidemiology, and End Results database. Cancer Manag Res 10: 6525-6535.

5. Baum RP, Hellwig D, Mezzetti M (2004) Position of nuclear medicine modalities in the diagnostic workup of cancer patients: lung cancer. Q J Nucl Med Mol Imaging 48(2): 119-142.

6. Lardinois D, Weder W, Hany TF, Kamel EM, Korom S, et al. (2003) Staging of non-small-cell lung cancer with integrated positron-emission tomography and computed tomography. N Engl J Med 348(25): 25002507.

7. Chen HH, Chiu NT, Su WC, Guo HR, Lee BF (2012) Prognostic value of whole-body total lesion glycolysis at pretreatment FDG PET/CT in nonsmall cell lung cancer. Radiology 264(2): 559-566.

8. Goodgame B, Pillot GA, Yang Z, Shriki J, Meyers BF, et al. (2008) Prognostic value of preoperative positron emission tomography in resected stage I non-small cell lung cancer. J Thorac Oncol 3(2): 130-134.
9. Hellwig D, Baum RP, Kirsch C (2009) FDG-PET, PET/CT and conventional nuclear medicine procedures in the evaluation of lung cancer: a systematic review. Nuklearmedizin 48(2): 59-69.

10. Vansteenkiste J, Fischer BM, Dooms C, Mortensen J (2004) Positronemission tomography in prognostic and therapeutic assessment of lung cancer: systematic review. Lancet Oncol 5(9): 531-540.

11. Benz MR, Herrmann K, Walter F, Garon EB, Reckamp KL, et al. (2011) (18)F-FDG PET/CT for monitoring treatment responses to the epidermal growth factor receptor inhibitor erlotinib. J Nucl Med 52(11): 16841689 .

12. Huang W, Zhou T, Ma L, Sun H, Gong H, et al. (2011) Standard uptake value and metabolic tumor volume of ${ }^{18} \mathrm{~F}$-FDG PET/CT predict shortterm outcome early in the course of chemoradiotherapy in advanced non-small cell lung cancer. Eur J Nucl Med Mol Imaging 38(9): 16281635.

13. Inal A, Kucukoner M, Kaplan MA, Urakci Z, Nas N, et al. (2013) Is (18) F-FDG-PET/CT prognostic factor for survival in patients with small cell lung cancer? Single center experience. Rev Port Pneumol 19(6): 260265 .

14. Kohutek ZA, Wu AJ, Zhang Z, Foster A, Din SU, et al. (2015) FDG-PET maximum standardized uptake value is prognostic for recurrence and survival after stereotactic body radiotherapy for non-small cell lung cancer. Lung Cancer 89(2): 115-120.

15. Lin Y, Lin WY, Kao CH, Yen KY, Chen SW, et al. (2012) Prognostic value of preoperative metabolic tumor volumes on PET-CT in predicting disease-free survival of patients with stage I non-small cell lung cancer. Anticancer Res 32(11): 5087-5091.

16. Mehta G, Chander A, Huang C, Kelly M, Fielding P (2014) Feasibility study of FDG PET/CT-derived primary tumour glycolysis as a prognostic indicator of survival in patients with non-small-cell lung cancer. Clin Radiol 69(3): 268-274.

17. Chen KN, Zhongguo Fei Ai Za Zhi (2016) Small Cell Lung Cancer and TNM Staging. 19(6): 409-412.

18. Olivier A, Petyt G, Cortot A, Scherpereel A, Hossein Foucher C (2014) Higher predictive value of tumour and node [18F]-FDG PET metabolic volume and TLG in advanced lung cancer under chemotherapy. Nucl Med Commun 35(9): 908-915.

19. Torre LA, Siegel RL, Jemal A (2016) Lung Cancer Statistics. Adv Exp Med Biol 893: 1-19.

20. Janne PA, Freidlin B, Saxman S, Johnson DH, Livingston RB, et al. (2002) Twenty-five years of clinical research for patients with limited-stage small cell lung carcinoma in North America. Cancer 95(7): 1528-1538.

21. Micke P, Faldum A, Metz T, Beeh KM, Bittinger F, et al. (2002) Staging small cell lung cancer: Veterans Administration Lung Study Group versus International Association for the Study of Lung Cancer--what limits limited disease? Lung Cancer 37(3): 271-276.

22. Al Sarraf N, Gately K, Lucey J, Aziz R, Doddakula K, et al. (2008) Clinical implication and prognostic significance of standardised uptake value of primary non-small cell lung cancer on positron emission tomography: analysis of 176 cases. Eur J Cardiothorac Surg 34(4): 892-897.

23. Dhital K, Saunders CA, Seed PT, O Doherty MJ, Dussek J (2000) [(18)F] Fluorodeoxyglucose positron emission tomography and its prognostic value in lung cancer. Eur J Cardiothorac Surg 18(4): 425-428.

24. Hanin FX, Lonneux M, Cornet J, Noirhomme P, Coulon C, et al. (2008) Prognostic value of FDG uptake in early stage non-small cell lung cancer. Eur J Cardiothorac Surg 33(5): 819-823.

25. Patz EF Jr, Connolly J, Herndon J (2000) Prognostic value of thoracic FDG PET imaging after treatment for non-small cell lung cancer. AJR Am J Roentgenol 174(3): 769-774.

26. Chung HW, Lee KY, Kim HJ, Kim WS, So Y (2014) FDG PET/CT metabolic tumor volume and total lesion glycolysis predict prognosis in patients 
with advanced lung adenocarcinoma. J Cancer Res Clin Oncol 140(1): 89-98.

27. Zhang H, Wroblewski K, Appelbaum D, Pu Y (2013) Independent prognostic value of whole-body metabolic tumor burden from FDG-PET in non-small cell lung cancer. Int J Comput Assist Radiol Surg 8(2): 181191.

28. Cerfolio RJ, Bryant AS, Ohja B, Bartolucci AA (2005) The maximum standardized uptake values on positron emission tomography of a non-

\section{ISSN: 2574-1241}

DOI: 10.26717/BJSTR.2019.18.003187

Huynh Quang H. Biomed J Sci \& Tech Res

This work is licensed under Creative Commons Attribution 4.0 License

Submission Link: https://biomedres.us/submit-manuscript.php small cell lung cancer predict stage, recurrence, and survival. J Thorac Cardiovasc Surg 130(1): 151-159.

29. Ginsberg MS, Grewal RK, Heelan RT (2007) Lung cancer. Radiol Clin North Am 45(1): 21-43.

30. Pan H, Shi X, Xiao D, He J, Zhang Y, et al. (2017) Nomogram prediction for the survival of the patients with small cell lung cancer. J Thorac Dis 9(3): 507-518.

\begin{tabular}{ll} 
BIOMEDICAL & Assets of Publishing with us \\
RESEARCHES & - Global archiving of articles \\
\hline & - Immediate, unrestricted online access \\
\hline
\end{tabular}

